\title{
Micropallet Arrays for the Capture, Isolation and Culture of Circulating Tumor Cells from Whole Blood of Mice Engrafted with Primary Human Pancreatic Adenocarcinoma
}

\author{
Philip C. Gach ${ }^{1}$, Peter J. Attayek ${ }^{2}$, Rebecca L. Whittlesey ${ }^{3}$, Jen Jen Yeh ${ }^{3,4,5}$, and Nancy L. \\ Allbritton ${ }^{1,2,3,5,{ }^{*}}$ \\ ${ }^{1}$ Department of Chemistry, University of North Carolina, Chapel Hill, North Carolina 27599 \\ ${ }^{2}$ Department of Biomedical Engineering, University of North Carolina, Chapel Hill, North Carolina \\ 27599 and North Carolina State University, Raleigh, North Carolina 27695 \\ ${ }^{3}$ Lineberger Comprehensive Cancer Center, University of North Carolina, Chapel Hill, North \\ Carolina 27599 \\ ${ }^{4}$ Department of Surgery, Division of surgical Oncology, University of North Carolina, Chapel Hill, \\ North Carolina 27599 \\ ${ }^{5}$ Department of Pharmacology, University of North Carolina, Chapel Hill, North Carolina 27599
}

\begin{abstract}
Circulating tumor cells (CTCs) are important biomarkers of cancer progression and metastatic potential. The rarity of CTCs in peripheral blood has driven the development of technologies to isolate these tumor cells with high specificity; however, there are limited techniques available for isolating target CTCs following enumeration. A strategy is described to capture and isolate viable tumor cells from whole blood using an array of releasable microstructures termed micropallets. Specific capture of nucleated cells or cells expressing epithelial cell adhesion molecules (EpCAM) was achieved by functionalizing micropallet surfaces with either fibronectin, Matrigel or antiEpCAM antibody. Surface grafting of poly(acrylic acid) followed by covalent binding of protein A/G enabled efficient capture of EpCAM antibody on the micropallet surface. MCF-7 cells, a human breast adenocarcinoma, were retained on the array surface with $90 \pm 8 \%$ efficiency when using an anti-EpCAM-coated array. To demonstrate the efficiency of tumor cell retention on micropallet arrays in the presence of blood, MCF-7 cells were mixed into whole blood and added to small arrays $\left(71 \mathrm{~mm}^{2}\right)$ coated with fibronectin, Matrigel or anti-EpCAM. These approaches achieved MCF-7 cell capture from $\unlhd 10 \mu \mathrm{L}$ of whole blood with efficiencies greater than $85 \%$. Furthermore, MCF-7 cells intermixed with $1 \mathrm{~mL}$ blood and loaded onto large arrays $\left(7171 \mathrm{~mm}^{2}\right)$ were captured with high efficiencies ( $\geq 97 \%$ ), could be isolated from the array by a laser-based approach and were demonstrated to yield a high rate of colony formation ( $\ 85 \%$ ) after removal from the array. Clinical utility of this technology was shown through the capture, isolation and successful culture of CTCs from the blood of mice engrafted with primary human pancreatic tumors. Direct capture and isolation of living tumor cells from blood followed by analysis or culture will be a valuable tool for cancer cell characterization.
\end{abstract}

(C) 2013 Elsevier B.V. All rights reserved.

"Corresponding author. Tel.: +1 919966 2291; fax: +1 919962 2388, nlallbri@ unc.edu (N.L. Allbritton).

Publisher's Disclaimer: This is a PDF file of an unedited manuscript that has been accepted for publication. As a service to our customers we are providing this early version of the manuscript. The manuscript will undergo copyediting, typesetting, and review of the resulting proof before it is published in its final citable form. Please note that during the production process errors may be discovered which could affect the content, and all legal disclaimers that apply to the journal pertain. 


\section{Keywords}

microfabrication; surface functionalization; cell array; circulating tumor cells; cell sorting; pancreatic cancer

\section{INTRODUCTION}

Advances in clinical technologies have revealed the significance of low abundance biomolecules or cells for monitoring patient health. Many studies have demonstrated the utility of a non-invasive, "liquid" biopsy towards tracking disease progression or patient health by quantifying low-concentration biomolecules in blood. ${ }^{1}$ Recent reports have hypothesized that circulating tumor cells (CTCs) may provide valuable information regarding the phenotype and metastatic behavior of an individual's primary tumor. ${ }^{2,3}$ Efficient strategies to isolate these low abundance cells from peripheral blood may enable novel clinical diagnostics as well as better understanding of cancer cell biology.

Many recent studies have sought to capture CTCs from the peripheral blood of patients or animal models with cancer. Magnetic-activated cell sorting (MACS) and fluorescenceactivated cell sorting (FACS) are the oldest methods in use for isolating CTCs. ${ }^{3,4}$, Unfortunately, MACS sorting is only capable of collecting CTCs based on their surface markers, rarely provides $100 \%$ collection efficiency and is plagued by contaminating cells such as lymphocytes and other nonspecifically captured cells. FACS is a commonly used technology for retrieving pure cells from a heterogeneous population. The low abundance of CTCs present in blood necessitates removal of erythrocytes prior to cell sorting and generally suffers from loss of CTCs as well as reduced cell viability. ${ }^{5}$ Many of the methods depending on surface expression of EpCAM may also suffer from poor CTC capture and identification as a result of down regulation of EpCAM as the cells undergo an epithelial to mesechymal evolution. ${ }^{6}$

The inefficiency of these technologies at isolating CTCs has spurred the development of a multitude of new capture technologies to sort and analyze CTCs. Microfluidic based devices for isolating CTCs enhance substrate-cell interactions by increasing the effective surface $\operatorname{area}^{7}$ or by generating chaotic cell mixing to increase collisional contact with the surface. ${ }^{8}$ Some technologies have allowed collection of CTCs following capture by employing laser pressure catapulting (LPC) or integrating a releasable antibody-substrate linker. ${ }^{9,18,21}$ Microfluidic devices have also achieved isolation of CTCs from blood by employing enrichment within microwell arrays, ${ }^{10}$ fluidic switching ${ }^{11}$, dielectrophoresis ${ }^{12}$ and inertial focusing. ${ }^{13}$ Size-selective microfilters have been developed in a variety of geometries to effectively capture CTCs from whole blood. ${ }^{14}$ These size-based collection strategies are able to capture and collect CTCs with excellent viability, however, they are unsuccessful at capturing small CTCs and also suffer from clogging by lymphocytes when high blood volumes are filtered. Though the tissue-cultured cells captured by the devices described above retain a high level of proliferation; few reports have successfully demonstrated culture of CTCs acquired from animal models and CTC culture from patient samples remains extremely challenging due the fragile nature of these cells. ${ }^{4,15,16}$

Our lab has previously demonstrated the utility of arrays of releasable elements microfabricated on glass substrates termed 'micropallets' for sorting single adherent cells. ${ }^{17}$ Micropallet technology has shown success at sorting single cells in a mixed cell population with low reagent requirements, high post sorting yield and excellent viability. Additionally, this platform has exhibited utility for isolating small numbers of target cells from mixed populations especially when the target cells are present in low abundance. ${ }^{17}$ 
Functionalization of the micropallet surfaces with extracellular matrices (ECM) or capture antibodies has previously been reported to provide pre-enrichment of target cells prior to sorting..$^{18,19}$ In this report, the potential for using micropallet arrays to isolate tumor cells directly from whole blood is examined. The capture efficiency was tested for MCF-7 cells mixed into whole blood and loaded directly onto micropallet arrays functionalized with fibronectin, Matrigel, or anti-EpCAM. Isolation of MCF-7 cells from whole blood followed by laser-based release and culture was assessed along with the sorting of tumor cells directly from the whole blood of mice bearing patient-derived pancreatic ductal adenocarcinoma.

\section{MATERIALS AND METHODS}

\section{Micropallet functionalization}

Micropallets were initially fabricated, as described in the supporting information, and functionalized with fibronectin or Matrigel. Micropallet surfaces were treated with various proteins to aid in cell capture and surface adhesion. Initially, micropallet arrays were sterilized by rinsing with $95 \%$ ethanol and dried in a sterile hood. Excess ethanol was removed with five rinses with phosphate buffered saline (PBS). For capture of adherent cells, the top surfaces of the micropallets on the array were coated with $1 \mathrm{~mL}$ of $25 \mu \mathrm{g} / \mathrm{mL}$ fibronectin in PBS for one hour at room temperature. For attachment of primary cells, micropallets were incubated with 1\% Matrigel (Matrix Growth Factor Reduced, BD Biosciences, Franklin Lakes, NJ) in complete media for at least one hour at $37^{\circ} \mathrm{C}$. Following surface coating, the array was rinsed $5 \times$ with PBS. Alternatively, micropallet tops were functionalized with anti-EpCAM antibody, as described in the supporting information (Figure S1).

\section{MCF-7 cell culture immediately before and after array-based isolation}

MCF-7 cells (a human breast adenocarcinoma cell line) were cultured in Dulbecco's modified Eagle's medium (DMEM) supplemented with fetal bovine serum (FBS) (10\%), Lglutamine $\left(584 \mathrm{mg} \mathrm{L}^{-1}\right)$, penicillin $\left(100\right.$ units $\left.\mathrm{mL}^{-1}\right)$ and streptomycin $\left(100 \mu \mathrm{g} \mathrm{ml}^{-1}\right)$ in a $37^{\circ} \mathrm{C}$ incubator with a $5 \% \mathrm{CO}_{2}$ atmosphere. To ascertain the capture efficiency of adherent cell lines from whole blood, MCF-7 cells were intermixed with sheep blood (BioChemed Services, Winchester, VA). The sample was then mixed with $2 \mathrm{~mL}$ (small arrays) or $10 \mathrm{~mL}$ (large arrays) of culture media and overlaid onto a fibronectin, Matrigel or anti-EpCAM functionalized micropallet array. The array was then transferred to an incubator for at least 2 $\mathrm{h}$ to permit the adherent cells to settle and attach to the substrate. Isolated MCF-7 cells were cultured in MCF-7-cell conditioned media.

\section{Culture of primary human CTCs on arrays}

Whole blood samples obtained from a PDX model of human pancreatic cancer were cultured in a manner similar to the MCF-7 cells with the following exceptions ${ }^{20}$. Blood from the mice was collected into a BD Microtainer ${ }^{\circledR}$ tube (BD Biosciences, Franklin Lakes, NJ) and then diluted with $10 \mathrm{~mL}$ of pancreatic-cell conditioned media and incubated on the micropallet arrays for $48 \mathrm{~h}$ in a $37^{\circ} \mathrm{C}$ incubator with a $5 \% \mathrm{CO}_{2}$ atmosphere. Isolated pancreatic CTCs were cultured in pancreatic-cell conditioned media, prepared as described in the Supporting Information. Prior to cell analysis and separation, the arrays were rinsed 5-10 times with PBS to selectively remove the blood components. All animal studies were approved by University of North Carolina at Chapel Hill Animal Care and Use Committee and comply with Nation Institute of Health guidelines. 


\section{Microscopy and micropallet isolation}

Following immunostaining of CTCs, as described in the supporting information, screening for CTCs over the large micropallet array was achieved with an MVX10 MacroView microscope (Olympus, Center Valley, PA) and motorized stage (ProScan III motorized stage system, H138A/C ProScan upright microscope stage, ProScan III motorized focus control, Prior Scientific Inc., Rockland, MA) paired with an ORCA-Flash4.0 camera (Hamamatsu, Bridgewater, NJ). Custom MATLAB software was used to analyze the acquired images in addition to controlling the microscope, stage, and camera. Imaging small micropallet arrays and micropallet release was performed on an inverted microscope (Eclipse TE300, Nikon, Melville, NY) combined with a pulsed Nd:YAG laser (532 nm, 5 ns Polaris II laser, New Wave Research, Fremont, CA). Released micropallets were then magnetically collected onto a multiwell plate as described previously. ${ }^{21}$ Quantitative inaccuracies inherent to spiking low numbers of MCF-7 for cell capture experiments were reduced by collecting the waste generated during array rinsing and immunostaining into a petri dish. Imaging of captured cells on the micropallet array and non-adherent MCF-7 cells collected into the waste container provided the total MCF-7 cell count. The quotient of these values provided accurate determination of MCF-7 cell capture efficiencies.

\section{RESULTS AND DISCUSSION}

\section{Micropallet functionalization with anti-EpCAM}

Anti-EpCAM antibodies are frequently utilized to capture CTCs since many epithelialderived tumors overexpress EpCAM. ${ }^{22}$ The possibility of coating micropallets by antibodies was previously demonstrated by the physical absorption of IgG and IgE. The efficiency of micropallet functionalization by physical absorption of anti-EpCAM was evaluated by incubating micropallets with $10 \mathrm{ug} / \mathrm{mL}$ fluorescein labeled anti-EpCAM. No statistical difference (two-sided $t$-test, $t(6), \mathrm{p}=0.823$ ) was observed between green fluorescence images taken of $1002 \mathrm{~F}$ photoresist coated with native anti-EpCAM $(1005 \pm 14)$ or fluorescein labeled anti-EpCAM $(954 \pm 199)$. A higher density of potential linkage sites was generated by grafting poly(acrylic acid) (PAA) onto the micropallet surface. ${ }^{23}$ Free amino group proteins were then covalently linked to the grafted PAA through carbodiimidemediated amide formation. Effective binding of biomolecules to the micropallet tops was demonstrated by covalently attaching fluorescein-labeled protein $\mathrm{A}$. The average green fluorescence of the fluorescein-protein A PAA-coated $\gamma \mathrm{Fe}_{2} \mathrm{O}_{3}-1002 \mathrm{~F}$ was $3351 \pm 241$, statistically greater than that for native $\mathrm{Fe}_{2} \mathrm{O}_{3}-1002 \mathrm{~F}(638 \pm 104)$ or native protein A-PAAcoated $\gamma \mathrm{Fe}_{2} \mathrm{O}_{3}-1002 \mathrm{~F}(852 \pm 9$ ) (one-way ANOVA, $\mathrm{F}(2,6)=98.86, \mathrm{p}=0.00003)$. For these experiments, the reaction time for PAA grafting $(12 \mathrm{~h})$ was greatly reduced compared to that reported previously $(84 \mathrm{~h})^{23}$ since the longer incubation times with cerium (IV) ammonium nitrate (CAN) destroyed the virtual air walls between the micropallets. The virtual air walls remained intact for the shorter duration reaction times although the PAA coating was reduced relative to that of the 84-h reaction protocol (data not shown). The extent and amount of anti-EpCAM antibody loaded onto the PAA grafted micropallets was measured by incubating native anti-EpCAM or fluorescein-labeled anti-EpCAM on $\gamma \mathrm{Fe}_{2} \mathrm{O}_{3}-1002 \mathrm{~F}$ grafted with protein A/G-coated PAA. A significant increase in the green fluorescence intensity was observed for $\gamma \mathrm{Fe}_{2} \mathrm{O}_{3}-1002 \mathrm{~F}$ coated with fluorescein-anti-EpCAM with respect to native anti-EpCAM or untreated $\gamma \mathrm{Fe}_{2} \mathrm{O}_{3}-1002 \mathrm{~F} ; 2120 \pm 185,1725 \pm 42$ and $638 \pm 104$, respectively (Figure 1) (one-way ANOVA, $F(2,6)=37.74, p=0.0004$ ). Functionalization of anti-EpCAM by attachment to PAA-protein A/G exhibited the greatest surface density of anti-EpCAM and so was employed for all subsequent experiments. Replacement of the virtual air walls by a more rugged microstructure boundary such as poly(ethylene glycol) (PEG) walls ${ }^{24}$ or polydimethylsiloxane (PDMS $)^{25}$ may permit prolonged incubation with 
the CAN solution and likely improve the quantity of anti-EpCAM immobilization to the array surfaces.

\section{Cell capture on micropallets coated with anti-EpCAM}

MCF-7 cells were utilized for initial assessment of cell capture by anti-EpCAM-coated micropallets. MCF-7 cells are a breast cancer cell line that exhibits a high expression of EpCAM antigen (509,500 molecules/cell), an order of magnitude higher than the EpCAM expression observed in most CTCs. ${ }^{26}$ In triplicate experiments, 10,000 MCF-7 cells were loaded onto the functionalized small micropallet arrays (12,769 micropallets) and the arrays were incubated for $10 \mathrm{~min}$ (Figure S2). Nine images of the arrays were acquired covering a fraction of the array's total area (2430 micropallets) and the cells in each image were counted. 1127 cells were identified (Fig. 2A). The arrays were then inverted and gently agitated to remove unbound and loosely attached MCF-7 cells. The arrays were imaged again and cells counted. $90 \pm 8 \%(\mathrm{n}=3)$ of the cells remained attached to the arrays (Fig. 2B).

The specificity of cell capture was then determined by repeating the experiment with HeLa cells ( $\mathrm{n}=3$ arrays, 2430 micropallets imaged, 988 total cells initially identified) (Fig. 2C). HeLa cells were used as a control since these cells express little to no EpCAM. ${ }^{27}$ Only $12 \pm$ $5 \%$ of the HeLa cells (118 total cells) were retained following inversion and agitation of the array (Fig. 2D). The significantly reduced collection of HeLa cells relative to MCF-7 cells suggested that the mechanism of cell capture for these short incubation times was primarily due to antibody-antigen binding as opposed to non-specific cell adhesion to the substrate (two-sided $t$-test, $t(6), \mathrm{p}=0.0001$ ).

The non-specific capture of cells was examined by monitoring the attachment of MCF-7 cells on grafted arrays not coated with anti-EpCAM, i.e. micropallets grafted with PAA and functionalized with only Protein A/G. Following a 10 min incubation period and array inversion, $21 \pm 11 \%$ of the MCF-7 cells remained adhered to the micropallets (316 cells). Repeating the control experiment with a prolonged incubation period of $2 \mathrm{~h}$ drastically enhanced the retention of MCF-7 to $87 \pm 4 \%$ (1456 adhered cells). These significantly different results imply the initial mechanism of cell attachment to anti-EpCAM functionalized micropallets is antibody-antigen binding (two-sided $t$-test, $t(6), \mathrm{p}=0.0006$ ) rather through an integrin-mediated mechanism. Enrichment of tumor cells by antibodybased binding ensures collection of the majority of antigen-expressing CTCs from a sample. In future studies, micropallet surfaces could be functionalized with other CTC binding molecules to capture cells with minimal EpCAM expression. ${ }^{6}$

\section{MCF-7 cell capture from whole blood with micropallets}

Enrichment of all or only viable CTCs has previously been demonstrated by selective capture of these cells onto an antibody ${ }^{7}$ or extracellular matrix ${ }^{28}$ coated substrate, respectively. Each capture strategy has its own advantages. For example, the majority of CTCs in circulation are either apoptotic or non-proliferating and thus may not be able to spontaneously adhere to surfaces using integrins and other ECM-binding molecules. ${ }^{29}$ These cells may need to be passively captured onto surfaces using high affinity moieties such as antibodies in order to provide a total CTC cell count. However, these selective techniques could potentially overlook capture of CTCs with unknown surface markers.

CTC enrichment directly from whole blood was examined by testing capture by micropallets with three different surface functionalizations: anti-EpCAM, fibronectin or Matrigel. As a proof-of-principle, approximately $100 \mathrm{MCF}-7$ cells were mixed into 1,10 or $100 \mu \mathrm{L}$ of whole blood which was then diluted with cell culture media $(2 \mathrm{~mL})$ and then loaded onto 
small arrays, in triplicate experiments (Figure 3, Table 1). For ease of analysis, arrays comprising 12,769 elements were utilized in place of the larger micropallet arrays (Figure S2). The quantity of blood added to the smaller arrays was equivalent (blood volume/array surface area) to adding $0.1,1$ and $10 \mathrm{~mL}$ of whole blood to the larger micropallet arrays used below. The arrays were placed in an incubator for a designated incubation period designed to capture a maximal number of target cells; 2-h for anti-EpCAM micropallets and 8-h for fibronectin and Matrigel-coated micropallets. These longer times were employed to enable the CTCs to migrate through the multilayered red blood cells to reach the pallet surface. Since capture of MCF-7 cells on negatively charged surfaces required much longer times than that for antibody-coated surfaces, longer incubation times were employed for the extracellular matrix-based capture.

Immediately prior to imaging, the arrays were rinsed with PBS to remove unattached cells. The efficiency of erythrocyte and lymphocyte removal was dependent on the number of rinses applied to the array. After 10 rinses, < 21000 erythrocytes and 220 lymphocytes remained on the arrays with the different tailored surfaces (Table 1). This is approximately 1 erythrocyte and 0.01 lymphocytes per micropallet, adequate enough to allow quality imaging of the micropallets (Figure 3). Of the MCF-7 cells mixed into 1,10 or $100 \mu \mathrm{L}$ whole blood, $>85 \%, \geq 87 \%$, and $>38 \%$ of the MCF-7 cells attached to the micropallets, respectively (Table 1). Interestingly, the capture efficiency of MCF-7 cells intermixed with blood was not significantly different for the anti-EpCAM, fibronectin or Matrigel-coated arrays despite contrasting capture mechanisms (one-way ANOVA, $[1 \mu \mathrm{L}$ blood $] \mathrm{F}(2,6)=$ $2.275, \mathrm{p}=0.184,[10 \mu \mathrm{L}$ blood $] \mathrm{F}(2,6)=1.076, \mathrm{p}=0.399,[100 \mu \mathrm{L}$ blood $] \mathrm{F}(2,6)=0.979, \mathrm{p}$ $=0.429$ ). The maximal capture efficiency of $96 \%$ corresponded to the percentage of living cells within a typical MCF-7 culture since the rapidly growing cells exhibit a high percentage of senescent cells (5\%). The low capture efficiency of MCF-7 cells when mixed into $100 \mu \mathrm{L}$ whole blood and incubated on the array under static conditions was likely due to the dense layer of erythrocytes on the array. When the volume of blood cells was sufficiently dilute, MCF-7 cells were able to encounter the array surface and adhere to the micropallets. Efficient CTC isolation from clinically relevant volumes of blood could potentially be achieved through lysis of erythrocytes or by use of larger arrays, as described below.

\section{Isolation and expansion of MCF-7 cells captured from whole blood}

A large array $(10.1 \times 7.1 \mathrm{~cm}, 1.3$ million micropallets $)$ was employed for determining the utility of micropallets for isolating CTCs directly from clinically relevant volumes of whole blood. Capture efficiencies were examined by mixing 1, 10 and $100 \mathrm{MCF}-7$ cells into $1 \mathrm{~mL}$ of whole blood diluted with $10 \mathrm{~mL}$ media and then incubated on the arrays. Since all surface coatings captured MCF-7 cells equally, we only employed fibronectin-coated micropallet arrays due to its ease of functionalization and low cost. Following removal of non-adherent cells by rinsing with PBS, MCF-7 cells were stained with Hoechst 33342 and fluoresceinlabeled anti-CD45 and Alexa Fluor 647-labeled anti-EpCAM antibody. MCF-7 cells (Hoechst $33342^{+} / \mathrm{CD}^{-} / \mathrm{EpCAM}^{+}$) were enumerated by imaging the arrays with an automated wide field microscope (Fig. 4A). A customized MATLAB image analysis program identified 2, 10 and 96 MCF-7 cells from the arrays loaded with the blood samples expected to have 1, 10 and 100 cells, respectively. The micropallet arrays enabled the systematic visualization of cells attached to the array over an extended time-frame prior to sorting. Isolation of MCF-7 cells from the array was achieved by laser-based release of 2, 10 and $20 \mathrm{MCF}-7$-containing micropallets followed by collection using a magnetic field, as described previously (Fig. 4B). ${ }^{21}$ After a 7-d incubation; 2 of 2, 10 of 10 and 17 of 20 of the collected cells on micropallets formed colonies demonstrating that the cells remained viable following mixing and incubation with the blood sample. All cell colonies were Hoechst 
$33342^{+}, \mathrm{EpCAM}^{+}$, and $\mathrm{CD}^{4} 5^{-}$indicating that the cells retained their initial phenotype (Fig. 4C).

\section{Isolation of CTCs from patient-derived xenograft (PDX) mouse models}

To further validate the efficacy of micropallet technology and demonstrate possible clinical significance, micropallets were employed to isolate CTCs from the blood of mice engrafted with human pancreatic ductal adenocarcinoma (PDAC) tumors (Fig. 5A). PDX mice have previously been shown to be excellent mimics of human disease and fully recapitulate the parental human tumor morphology and growth characteristics including subsequent extravasation and metastases formation. ${ }^{30}$ The small volumes of blood $(<1 \mathrm{~mL})$ and CTCs $(<100)$ in these mice make analysis and culture of CTCs from individual mice by typical low-efficiency techniques ineffective. Whole blood collected from a PDX mouse $(0.6-1.0$ $\mathrm{mL}$ ) was mixed with pancreatic-cell conditioned media $(10 \mathrm{~mL})$ supplemented with insulin growth factor (IGF) and epithelial growth factor (EGF), and then incubated for $48 \mathrm{~h}$ on separate large micropallet arrays coated with Matrigel $(n=3)$. Matrigel, pancreatic-cell condition media and longer incubation periods were used to improve the chances of successfully culturing CTCs on the micropallets. Non-adherent cells were then washed from the array with PBS. Incubation of the arrays with blood for $48 \mathrm{~h}$ increased lymphocyte capture 4.4-fold relative to the 8-hour incubation time. The longer incubation did not alter the numbers of captured erythrocytes. Arrays were stained with Hoechst 33342, fluoresceinanti-CD45 and Alexa Fluor 647-anti-Cytokeratin antibodies and CTCs enumerated by imaging as described above. With this approach, $13 \pm 6$ cells identified on the micropallets were Hoechst $33342^{+} / \mathrm{CD}^{-} 5^{-} /$Cytokeratin $^{+}$. This CTC count is similar to the value of $23 \pm 7$ CTCs per $\mathrm{mL}$ blood obtained from the same PDX model by Soper and colleagues using a microfluidic device (two-sided $t$-test, $t(7), \mathrm{p}=0.1048$ ). ${ }^{31}$

In addition to CTC enumeration, micropallet technology offers the capability of subsequent isolation and culture of viable CTCs. EpCAM has previously been demonstrated as being highly expressed in pancreatic adenocarcinoma, thus making it an excellent biomarker for viable detection of pancreatic adenocarcinoma-derived CTCs in contrast to the Cytokeratin which requires cell fixation. ${ }^{32}$ CTCs were captured from $0.7 \mathrm{~mL}$ PDX mouse blood and immunostained for EpCAM and CD45. Ten cells were identified on the arrays as being $\mathrm{EpCAM}^{+} /$Hoechst $33342^{+} / \mathrm{CD} 45^{-}$(Fig. B,C). The ten micropallets carrying CTCs were then released from the array and magnetically collected onto a multiwell plate coated with Matrigel. The cells remained adherent to the micropallets after isolation as observed by brightfield and fluorescence microscopy (Fig. 5D,E). Two of the ten cells isolated were viable as evidenced by their ability to form a cell colony following 7 days culture in pancreatic-cell conditioned media supplemented with IGF and EGF (Fig. 5F,G). The successful ex vivo colony formation from a minute quantity of CTCs was a result of the minimal sample processing and cellular manipulation afforded by micropallet technology. Successful culture of CTCs directly from the blood of xenograft mice models of human pancreatic adenocarcinoma will enable a better understanding of the biology of CTCs as well as the diversity in CTC properties.

\section{CONCLUSIONS}

This current work demonstrates the capability of tumor cell isolation directly from whole blood using the micropallet technology. Micropallets functionalized with either fibronectin or anti-EpCAM were able to efficiently capture MCF-7 cells from whole blood with high efficiency ( $\geq 85 \%$ ) and very minimal sample processing. In addition to capture and enumeration, MCF-7 cells could be isolated and cultured with a high success rate of colony formation ( $₫ 85 \%$ ). Furthermore, it was to capture, isolate and subsequently culture 
pancreatic CTCs derived from PDX mice. Technologies capable of isolating and culturing primary CTCs could play an essential role in our understanding of cancer metastasis as well as in drug development to prevent these events. While static conditions lead to low capture efficiencies with larger volumes of blood, micropallet bases with high aspect ratios and surfaces grafted with anti-EpCAM could be incorporated into a microfluidic channel increasing the volume of blood processed by these arrays. A combined micropalletmicrofluidic device might take advantage of the high throughput cell capture rates offered by microfluidics and the gentle release of micropallets holding captured CTCs. Additionally, previous successes of micropallets for sampling of cell colonies could be adapted to provide minimally invasive colony sampling and analysis over the lifespan of the developing tumor with results compared to tumor growth in vivo.

\section{Supplementary Material}

Refer to Web version on PubMed Central for supplementary material.

\section{Acknowledgments}

Yuli Wang, Chris Sims, Jonathan Clark, Pavak Shah, Gabriela Herrera and Jadwiga Smyla are thanked for providing technical support, advice, and suggestions. We thank Chapel Hill Analytical and Nanofabrication Laboratory (CHANL) for providing access to the facility's instrumentation. We thank Charlene Ross and the Animal Studies Core Facility for excellent technical assistance. This research was supported by the NIH (EB012549, CA139599, and CA140424).

\section{References}

1. Rissin D, Kan C, Campbell T, Howes S, Fournier D, Song L, Piech T, Patel P, Chang L, Rivnak A, Ferrell E, Randall J, Provuncher G, Walt D, Duffy D. Nat Biotechnol. 2010; 28:595. [PubMed: 20495550]

2. Cohen S, Punt C, Iannotti N, Saidman B, Sabbath K, Gabrail N, Picus J, Morse M, Mitchell E, Miller M, Doyle G, Tissing H, Terstappen L, Meropol N. J Clin Oncol. 2008; 26:3213. [PubMed: 18591556]

3. Cristofanilli M, Budd G, Ellis M, Stopeck A, Matera J, Miller M, Reuben J, Doyle G, Allard W, Terstappen L, Hayes D. N Engl J Med. 2004; 351:781. [PubMed: 15317891]

4. Ameri K, Luong R, Zhang H, Powell A, Montgomery K, Espinosa I, Bouley D, Harris A, Jeffrey S. Br J Cancer. 2010; 102:561. [PubMed: 20051957]

5. Takao M, Takeda K. Cytometry A. 2011; 79A:107. [PubMed: 21246706]

6. Gorges T, Tinhofer I, Drosch M, Rose L, Zollner T, Krahn T, von Ahsen O. BMC Cancer. 2012; 12

7. Nagrath S, Sequist LV, Maheswaran S, Bell DW, Irimia D, Ulkus L, Smith MR, Kwak EL, Digumarthy S, Muzikansky A, Ryan P, Balis UJ, Tompkins RG, Haber DA, Toner M. Nature. 2007; 450:1235. [PubMed: 18097410]

8. Wang S, Liu K, Liu J, Yu Z, Xu X, Zhao L, Lee T, Lee E, Reiss J, Lee Y, Chung L, Huang J, Rettig M, Seligson D, Duraiswamy K, Shen C, Tseng H. Angew Chem Int Ed. 2011; 50:3084.

9. Helzer KT, Barnes HE, Day L, Harvey J, Beilings PR, Forsyth A. Cancer Res. 2009; 69:7860. [PubMed: 19789350]

10. Kang JH, Krause S, Tobin H, Mammoto A, Kanapathipillai M, Ingber DE. Lab Chip. 2012; 12:2175. [PubMed: 22453808]

11. Schiro PG, Zhao M, Kuo JS, Koehler KM, Sabath PDE, Chiu PDT. Angew Chem Int Ed. 2012; 51:4618.

12. Gascoyne P, Noshari J, Anderson T, Becker F. Electrophoresis. 2009; 30:1388. [PubMed: 19306266]

13. Hur S, Mach A, Di Carlo D. Biomicrofluidics. 2011; 5

14. Zheng S, Lin H, Lu B, Williams A, Datar R, Cote R, Tai Y. Biomed Microdevices. 2011; 13:203. [PubMed: 20978853] 
15. Glinskii A, Smith B, Jiang P, Li X, Yang M, Hoffman R, Glinsky G. Cancer Res. 2003; 63:4239. [PubMed: 12874032]

16. Glinsky GV, Glinskii AB, Berezovskaya O, Smith BA, Jiang P, Li XM, Yang M, Hoffman RM. Cell Cycle. 2006; 5:191. [PubMed: 16357534]

17. Wang Y, Young G, Bachman M, Sims C, Li G, Allbritton N. Anal Chem. 2007; 79:2359. [PubMed: 17288466]

18. Gunn N, Bachman M, Li G, Nelson E. J Biomed Mater Res, Part B. 2010; 95A:401.

19. Shadpour H, Sims CE, Allbritton NL. Cytometry A. 2009; 75A:609. [PubMed: 19504569]

20. Wang J, Geddings J, Aleman M, Cardenas J, Chantrathammachart P, Williams J, Kirchhofer D, Bogdanov V, Bach R, Rak J, Church F, Wolberg A, Pawlinski R, Key N, Yeh J, Mackman N. Blood. 2012; 119:5543. [PubMed: 22547577]

21. Gach P, Sims C, Allbritton N. Biomaterials. 2010; 31:8810. [PubMed: 20719380]

22. Went P, Lugli A, Meier S, Bundi M, Mirlacher M, Sauter G, Dirnhofer S. Hum Pathol. 2004; 35:122. [PubMed: 14745734]

23. Wang Y, Pai JH, Lai HH, Sims CE, Bachman M, Li GP, Allbritton NL. J Micromech Microeng. 2007; 17:1371.

24. Wang Y, Salazar GT, Pai JH, Shadpour H, Sims CE, Allbritton NL. Lab Chip. 2008; 8:734. [PubMed: 18432343]

25. Gach P, Wang Y, Phillips C, Sims C, Allbritton N. Biomicrofluidics. 2011; 5

26. Rao C, Chianese D, Doyle G, Miller M, Russell T, Sanders R, Terstappen L. Int J Oncol. 2005; 27:49. [PubMed: 15942643]

27. Sekine J, Luo S, Wang S, Zhu B, Tseng H, Yu H. Adv Mater. 2011; 23:4788. [PubMed: 21954025]

28. Paris P, Kobayashi Y, Zhao Q, Zeng W, Sridharan S, Fan T, Adler H, Yera E, Zarrabi M, Zucker S, Simko J, Chen W, Rosenberg J. Cancer Lett. 2009; 277:164. [PubMed: 19162393]

29. Chambers A, Groom A, MacDonald I. Nat Rev Cancer. 2002; 2:563. [PubMed: 12154349]

30. Patsialou A, Wang Y, Lin J, Whitney K, Goswami S, Kenny P, Condeelis J. Breast Cancer Res. 2012; 14

31. Jackson JM, Witek MA, Hupert ML, Brady C, Pullagurla S, Kamande J, Aufforth RD, Tignanelli CJ, Torphy RJ, Yeh JJ, Soper SA. Lab on a Chip. 2014

32. Fong D, Steurer M, Obrist P, Barbieri V, Margreiter R, Amberger A, Laimer K, Gastl G, Tzankov A, Spizzo G. J Clin Pathol. 2008; 61:31. [PubMed: 16775119] 


\section{Highlights}

- CTCs were captured from whole blood on an array.

- The array elements were releasable enabling viable CTC isolation.

- Greater than $85 \%$ of tumor cells were captured from $1 \mathrm{~mL}$ of whole blood.

- CTCs were captured and cultured from a xenografted human pancreatic adenocarcinoma. 

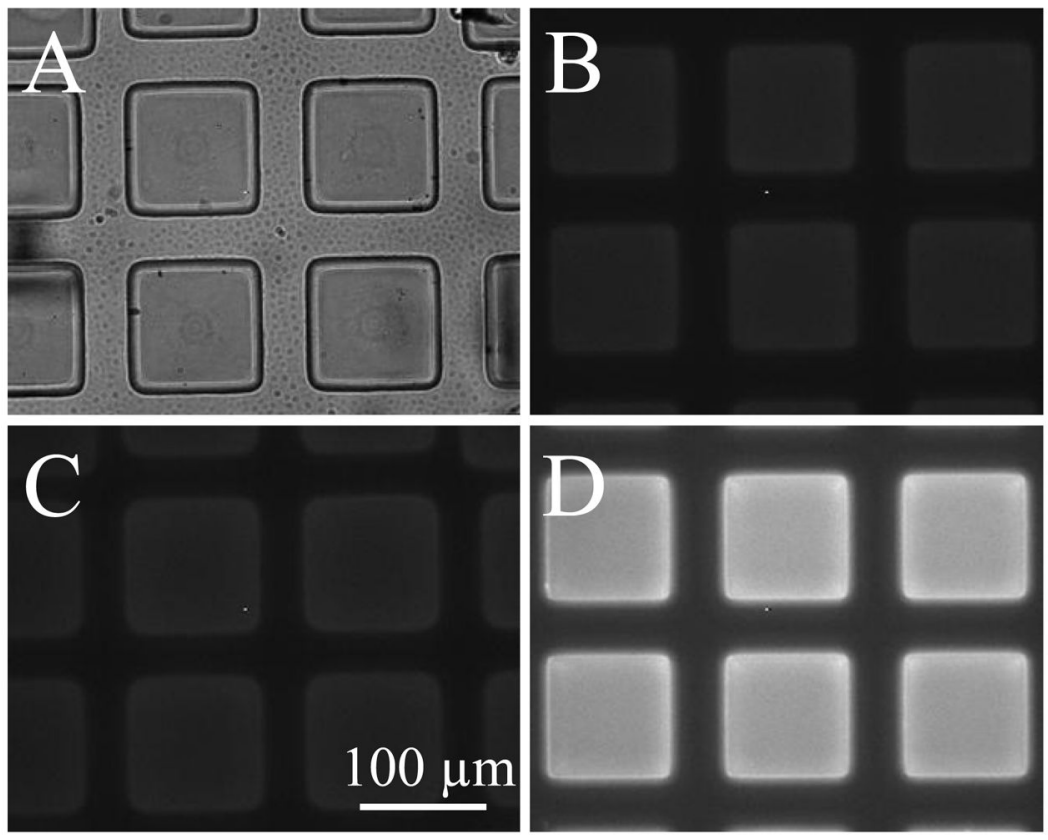

Figure 1.

anti-EpCAM functionalization of micropallets. Brightfield image of micropallets (A) and fluorescence image of native micropallets (B), fluorescein-labeled anti-EpCAM physically absorbed to micropallets (C) and fluorescein-labeled anti-EpCAM attached to micropallets using PAA-protein A/G (D). 

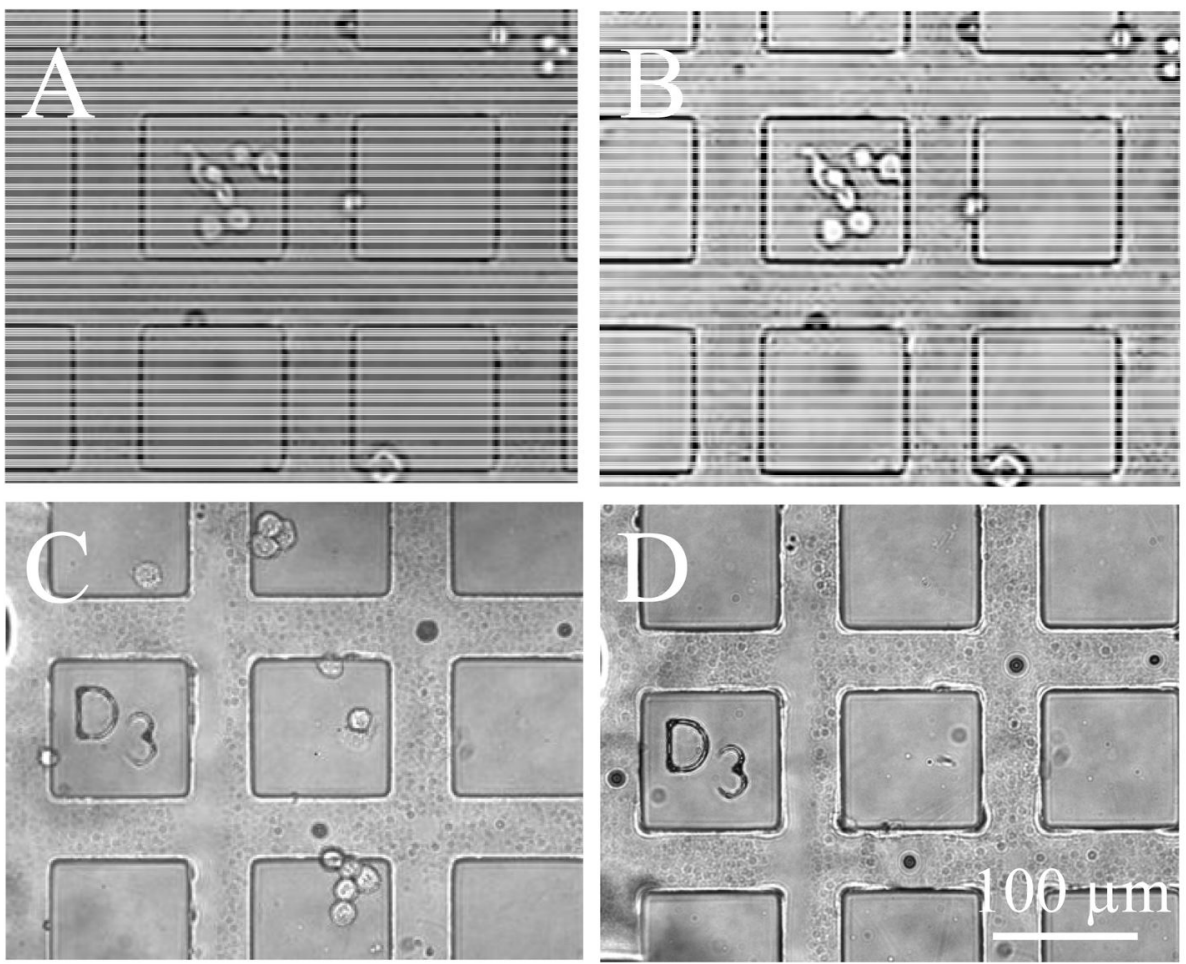

Figure 2.

Selective cell capture on anti-EpCAM functionalized micropallets. MCF-7 cells on an antiEpCAM functionalized micropallet array before (A) and after rinsing (B). HeLa cells on an anti-EpCAM functionalized micropallet array before (C) and after rinsing (D). 

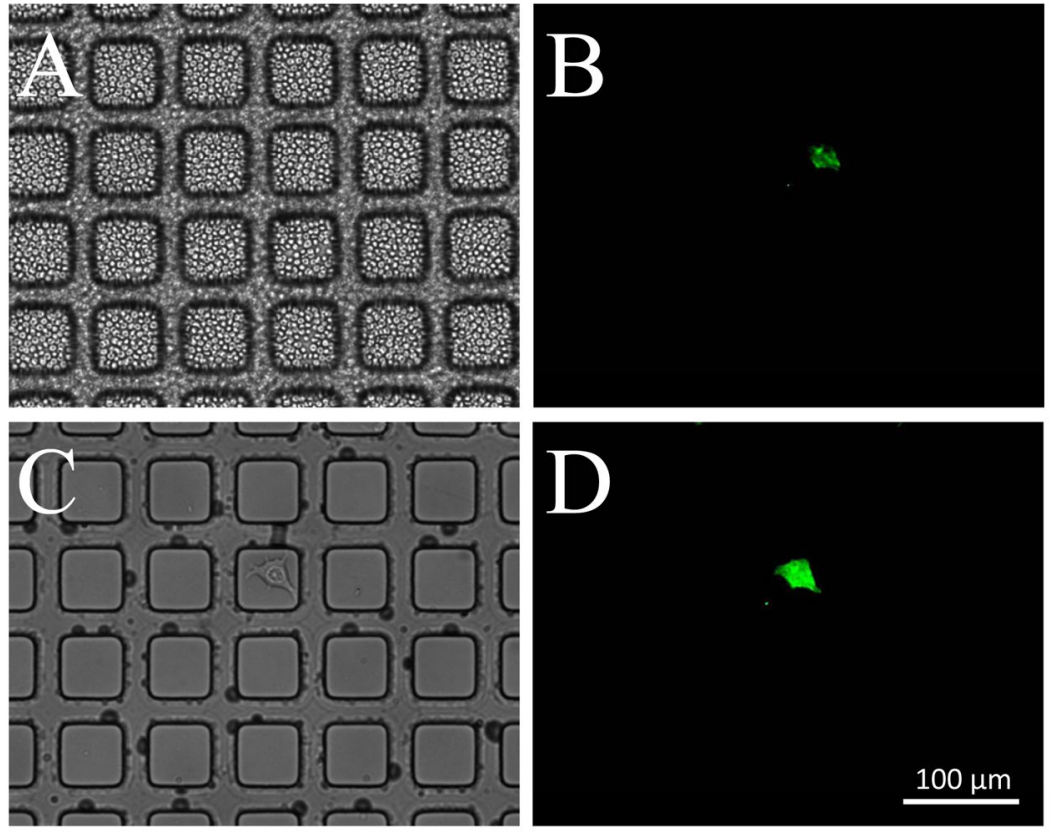

Figure 3.

Capture of MCF-7 cells from peripherial blood. Brightfield (A) and fluorescence images (B) of fibronectin-coated arrays overlaid with $10 \mu \mathrm{L}$ whole blood containing MCF-7 cells stained with fluorescein-labeled anti-EpCAM prior to removal of the blood. Brightfield (C) and fluorescence images (D) of the same array in A and B after sequential PBS rinses. 


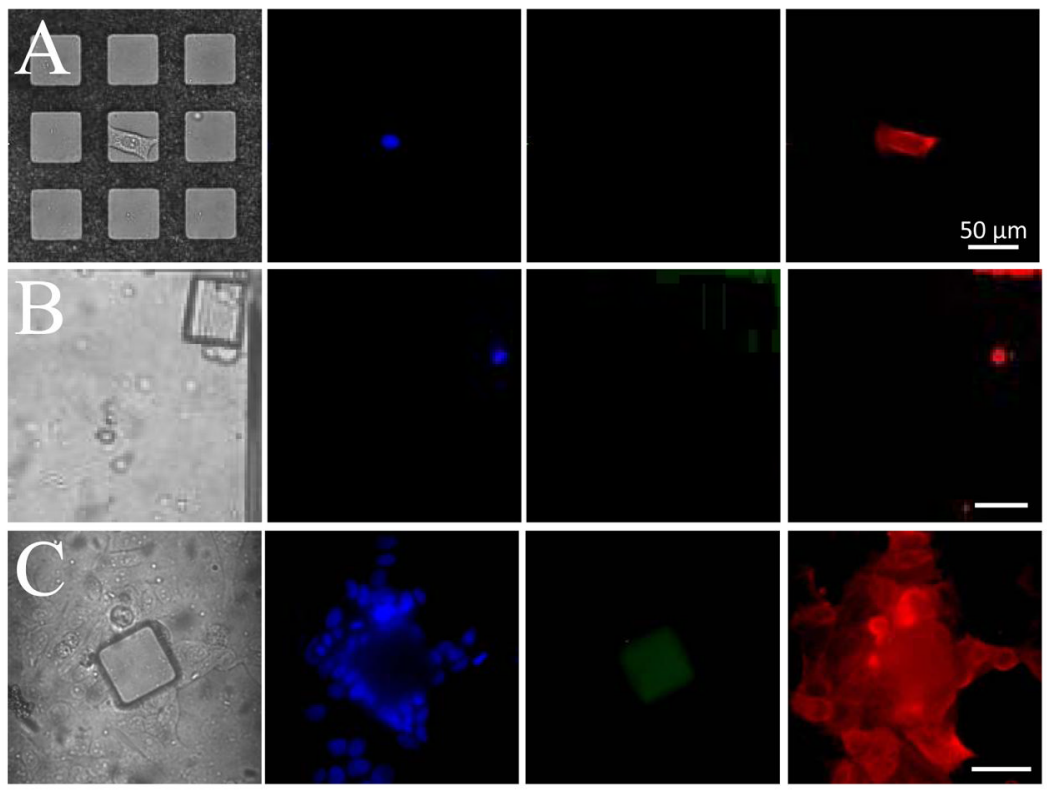

\section{Figure 4.}

MCF-7 cell isolation from peripherial blood and culture. Brightfield (left column) and fluorecence (right 3 columns) micrographs of a single MCF-7 cell identified after addition of $1 \mathrm{~mL}$ of whole blood to a large micropallet array (A). The cell in panel A immediately after micropallet release from the array and collection (B) and after 7 days of culture (C). The DNA of MCF-7 cells was stained with Hoechst 33342 nuclear stain (second column from left, blue). The cells were aso stained with Alexa Fluor 647-anti-EpCAM antibody (far right column, red) and fluorescein-anti-CD45 (second column from right, green). Scale bars represent $50 \mu \mathrm{m}$. 

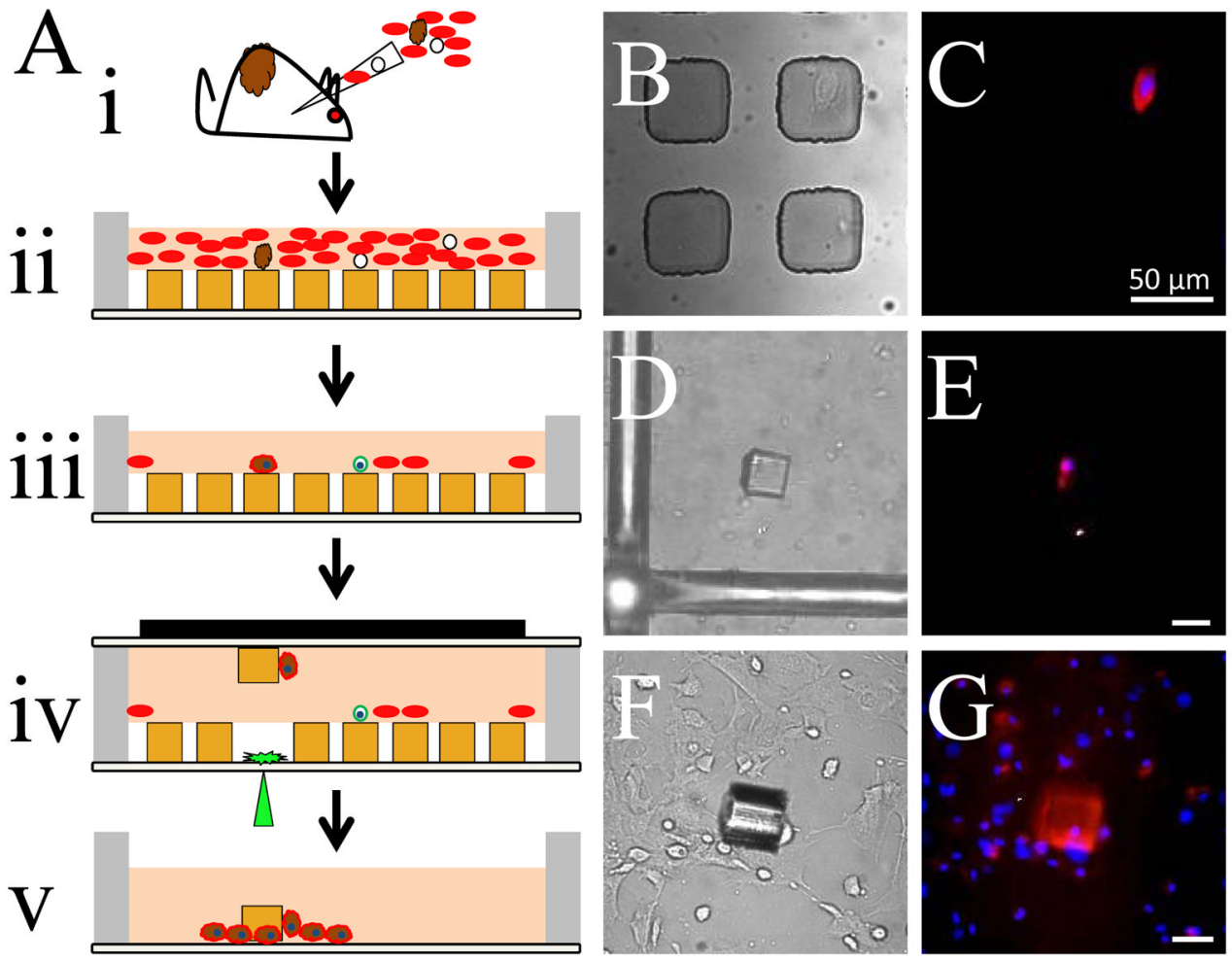

Figure 5.

Isolation and culture of CTCs from peripheral blood samples from a pancreatic cancer patient-derived xenograft. (A) Schematic of the collection of whole blood from a mouse implantated with a patient tumor (i), loading of blood onto micropallet arrays (ii), removal of non-adherent blood cells and immunostaining (iii), selective release and collection of target micropallets (iv) and CTC culture (v). (B,C) Whole blood was incubated on the array for 2 days and then the array washed to removed nonadherent cells. Shown are a brightfield (B) and a composite fluorescence image (Hoeschst 33342, anti-CD45, anti-EpCAM) (C) of a cell on the array. (D,E) A micropallet with a CTC was isolated from the array and immediately imaged as in panels B and C. The white dot below the CTC in E represents a fixed camera pixel. $(\mathrm{F}, \mathrm{G})$ A collected micropallet with a CTC was cultured for $7 \mathrm{~d}$, immunostained and imaged as in panels B and C. Scale bars represent $50 \mu \mathrm{m}$. 


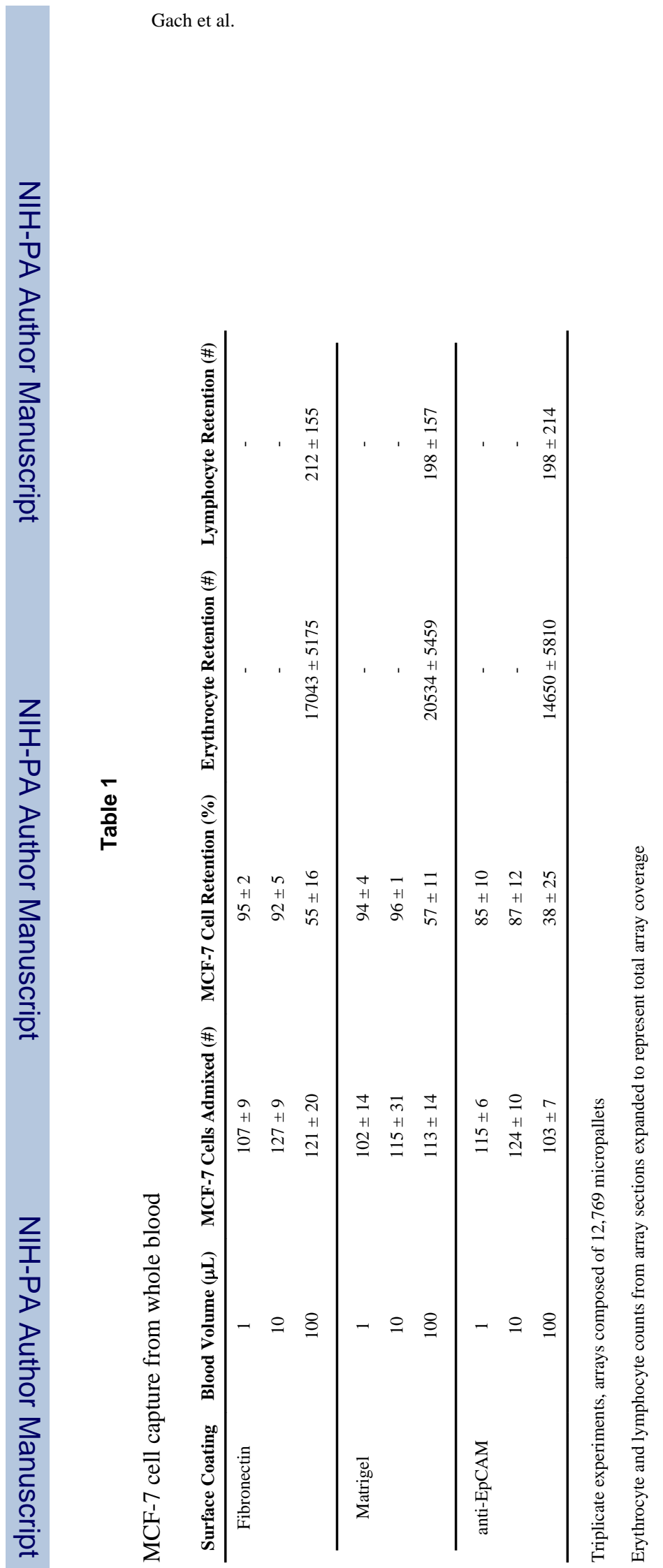

Page 16

Biosens Bioelectron. Author manuscript; available in PMC 2015 April 15. 